\title{
LA SEXUALIDAD EN EL DESARROLLO: HACIA UNA VISIÓN INCLUSIVA
}

\author{
Guillermo Núñez Noriega \\ Centro de Investigación en Alimentación y Desarrollo, A.C.
}

Patricia Ponce

Centro de Investigaciones y Estudios Superiores en Antropología Social (CIESAS)

\author{
Laura Woolfolk \\ Centro de Investigación en Alimentación y Desarrollo, A.C.
}

\section{RESUMEN}

En este artículo se presenta una reflexión informada sobre la evolución del concepto de "desarrollo" hasta la inclusión de la sexualidad, una dimensión de la vida personal y social. Se reflexiona sobre la importancia de esta inclusión, pero también sobre las limitaciones que presenta. Se considera, asimismo, que el planteo teórico queer permite superar estas limitaciones y sentar bases nuevas y sólidas para una concepción del desarrollo que tome en cuenta la sexualidad humana en su diversidad.

\section{PALABRAS CLAVE}

Desarrollo, Género, Sexualidad, Teoría queer

\section{ABSTRACT}

In this article we present an informed reflection on the evolution of the concept of "development" to the inclusion of sexuality, a dimension of personal and social life. We reflect on the importance of this inclusion, but also on the limitations it presents. We believe queer theory allows us to overcome these limitations and set a new and solid ground for a concept of development that take into account human sexuality in its diversity.

\section{KEY WORDS}

Development, Gender, Sexuality, Queer Theory 


\section{INTRODUCCIÓN}

El concepto de "desarrollo" forma parte de esas narrativas maestras ("metanarrativas" o "metarrelatos") 1 de la modernidad, al lado de otras como "civilización" y "progreso", que han tenido un impacto profundo y extenso en la vida de las personas y de los pueblos (Lyotard, 1979). En la medida de que parte de una metanarrativa, el concepto de desarrollo es muy importante en nuestros días pues se ha vuelto paradigmático para entender, organizar y justificar la política pública regional, nacional e internacional, con relevantes consecuencias, incluso, para la conceptualización que el sujeto hace de sí mismo, de su propia identidad y de su vida cotidiana. ${ }^{2}$ La teoría social contemporánea, particularmente a partir de su giro postmoderno, ha dado cuenta

1 Por metanarrativa, narrativa maestra o metarrelato, entendemos, siguiendo a Lyotard, un discurso que ofrece un sentido totalizador, trascendente o universal de la historia. En el caso de las metanarrativas de la modernidad, éstas ofrecen una visión del cambio social, cultural, económico o político de las sociedades humanas, como un camino "evolutivo" o "ascendente" llamado "civilización", "progreso", "desarro1lo". El principio fundamente de este cambio es en la modernidad, la Razón, o el uso de la razón, como capacidad humana, para generar un conocimiento científico del entorno natural y social, y su aplicación en procesos tecnológicos, organizativos y administrativos que harían posible un cambio ordenado hacia "etapas superiores" o más "avanzadas" que las anteriores.

2 Los criterios para "medir" ese "avance", planteado por estos discursos maestros del cambio social, requieren necesariamente de una elección valorativa que no siempre se hace consciente o se discute, sino que más bien se acepta como evidente por sí misma, pero que puede ir desde los usos y costumbres en la mesa, como señala Norbert Elias (2009), para el caso del concepto civilización, hasta un camino pavimentado, para el caso de la noción de progreso en la historia de Sonora (Nuñez, 1993), o los materiales de construcción del piso de una vivienda, para el caso del concepto desarrollo, tal y como lo plantean las políticas gubernamentales actuales en México. del origen, las características e implicaciones del horizonte cultural $^{3}$ de la modernidad, en el cual estamos inmersos, haciéndonos conscientes de su historicidad y de sus efectos de poder y libertad. ${ }^{4}$

Arraigado en la promesa moderna de que el uso de la razón mejoraría la condición humana y la vida en sociedad, el concepto de desarrollo, heredero de nociones como civilización, de uso creciente desde el siglo XVI, o de progreso, en boga en el siglo XIX y principios del siglo XX, se ha enriquecido desde su aparición a mediados del siglo pasado. De una definición restringida a la de "crecimiento económico", que tuvo en sus inicios, el concepto desarrollo ha transitado por definiciones que incluyen al ser humano y sus necesidades básicas, así como por novedosas reflexiones sobre el medio ambiente, la equidad, la justicia social y la democracia. Las feministas, con su trabajo de visualización de las condiciones de las mujeres en las sociedades y su relación a las políticas de desarrollo, así como con sus conceptos de género, equidad, inclusión y vulnerabilidad, entre otros, han tenido un papel destacado en esta transformación cognitiva, sociocultural y política vinculada al concepto que nos ocupa.

3 Retomamos el concepto de horizonte cultural del teórico norteamericano marxista Frederic Jameson (1981) para referirnos a la modernidad. Para ver una aplicación de este concepto véase el artículo "La metanarrativa de progreso y la emergencia de subalternidades: el caso de "la sierra" en Sonora, México" (Núñez, 1993), que ayuda a entender los procesos de modernización regional en el estado de Sonora y su dimensión territorial.

4 Sobre el tema de la modernidad y sus implicaciones de poder se ha escrito mucho. Baste referir aquí a los planteamientos de Michel Foucault sobre las tecnologías de saber-poder modernos (Foucault, 2012) y los planteamientos sobre la modernidad, el poder y la libertad de Zygmut Bauman (1992). 
En los últimos cincuenta años, la sexualidad se ha convertido en un tema importante de la disputa sociocultural y política, y ha dado lugar al surgimiento de reivindicaciones ciudadanas y movimientos sociales regionales, nacionales y globales. La diversidad de reivindicaciones se ha agrupado en conceptos como el de "Derechos Sexuales y Reproductivos". Asimismo, las ciencias sociales han elaborado interesantes reflexiones teóricas y conceptuales que muestran la centralidad de la sexualidad en la organización de las relaciones de poder modernos. ${ }^{5}$

A finales del siglo XX, en el marco de la Conferencia Internacional sobre Población y Desarrollo (CIPyD), se incorporó el tema de la sexualidad en la agenda del desarrollo global, subordinada al tema de la población y sus preocupaciones por la salud reproductiva y la equidad de género, entendida como equidad entre hombres y mujeres. Sin embargo, desde la perspectiva teórica queer - que cuestiona el sistema de homologías de sexo, género y orientación sexual basado en binarismos excluyentes, y que se articula desde finales de los años ochenta del siglo XX como producto de la confluencia del feminismo, el postestructuralismo, la respuesta social a la epidemia del sida y la discusión interna del movimiento LGBTTI (Vance, 1989; De Lauretis, 1993; Butler, 1990, 1993; Jagose 1996)_, esta incorporación es insuficiente.

5 La producción y los autores son numerosos, remitimos sin embargo a los trabajos clásicos de Michel Foucault, Jeffrey Weeks, Anne Fausto-Sterling, David Halperin y Judith Butler, por mencionar sólo algunos ampliamente reconocidos.

\section{APROXIMACIONES AL CONCEPTO} DE DESARROLLO

A través del tiempo, el término desarrollo se ha definido de diferentes maneras y ha tenido diversos impactos en los ejes rectores que determinan la conducción de los gobiernos en materia política, económica, social, cultural y ambiental, así como en la implementación de políticas públicas que buscan promover el desarrollo de un país o de una región. Los ejes rectores que determinan la conducción o la acción para el establecimiento de políticas públicas en materia de desarrollo son dinámicos y transitorios, esto es, evolucionan de acuerdo a los valores dominantes de la sociedad, a la praxis en la aplicación de modelos en países desarrollados, a las nuevas corrientes de pensamiento en las disciplinas sociales y a los intereses de los organismos mundiales de financiamiento y de las agencias de cooperación para el desarrollo.

No existe una definición de desarrollo única y permanente a lo largo del tiempo puesto que se trata de una construcción social e histórica y, por ende, dinámica (Colmegna e Isla, 2007). Definir el concepto de desarrollo, por lo tanto, es algo complejo y requiere, para ello, que se considere la historia misma del concepto, las reflexiones académicas y las luchas sociales que le han ido dando forma. Es imposible detallar aquí la relación entre las condiciones materiales e ideológicas de vida y la evolución misma del concepto de desarrollo. Valga mencionar de inicio, sin embargo, que el concepto desarrollo se ha asociado a diversos términos, tales como: crecimiento económico, distribución, igualdad, 
sustentabilidad, calidad de vida, bienestar, capacidades y desarrollo humano, entre otros.

El concepto de desarrollo puede vincularse con nociones sociopolíticas antiguas de cambio y mejoramiento social, ya presentes en la Grecia clásica incluso, pero su acepción actual hunde sus raíces en la modernidad y en conceptos previos como civilización y progreso. No obstante, los estudiosos coinciden en señalar que no fue sino hasta 1949 cuando el término adquirió legitimidad política. El evento fue el discurso inaugural del presidente norteamericano Harry Truman, quien lo utilizó para hacer referencia a regiones del planeta consideradas "atrasadas". De esta manera, el término de desarrollo comenzó a ser utilizado para aludir a las diferencias socioeconómicas entre países ricos, mientras que su contraparte, el subdesarrollo, se comenzó a emplear para referirse a los países pobres. (González, Bergesio y Golovanevsky, 2014)

La distinción entre países ricos y países pobres ha sido evidente a lo largo de la historia, pero el interés por el desarrollo es relativamente reciente: su concepto nace tras la Segunda Guerra Mundial. Jorge Rionda señala que la noción de desarrollo fue tratada por primera vez en documentos públicos en $1941^{6}$ y se ratificó en la Conferencia de San Francisco en 1945. ${ }^{7}$ En el

6 El 14 de agosto de 1941, el presidente de Estados Unidos, Franklin Delano Roosevelt, y el primer ministro del Reino Unido, Winston Churchill, propusieron una serie de principios para la colaboración internacional con el objetivo de mantener la paz y la seguridad. El documento, firmado durante la reunión mantenida a bordo del HMS Prince of Wales "en algún lugar en alta mar", se conoce con el nombre de Carta del Atlántico (ONU, 2014).

7 El primero de enero de 1942, representantes de 26 naciones aliadas que lucharon contra las Potencias del Eje, periodo comprendido entre finales de los años cuarenta y hasta finales de los años cincuenta el objetivo fundamental del desarrollo era el crecimiento económico (Rionda, 2005). Según Luz Alicia Cárdenas (1998), el desarrollo fue entendido durante el periodo de 1940 a 1960 como como un sinónimo de crecimiento económico que utilizaba el Producto Interno Bruto (PIB) y, sobre todo, el PIB per cápita, como medida corriente del nivel de desarrollo que se registraba en una región o país. Bajo esta noción, se elaboró una política de "modernización", ${ }^{8}$ emprendida por los países subdesarrollados, que tenía como finalidad crear un proceso acelerado de industrialización con la promesa de un mayor bienestar social.

No obstante, las políticas de desarrollo centradas en el objetivo de incrementar el Producto Interno Bruto (PIB) a través de un proceso acelerado de industrialización, no trajeron consigo las "mejoras" esperadas, o no del todo, pues esas "mejoras" o "bienestar" sólo se llevaban a cabo en ciertas regiones o sectores sociales y dejaban al margen del proceso de "desarrollo" a muchos otros sectores y, por tanto, a millones de personas.

se reunieron en Washington D.C. para reafirmar su apoyo a la Carta Atlántica mediante la firma de la "Declaración de las Naciones Unidas". Este documento contenía el primer uso oficial del término "Naciones Unidas", propuesto por el presidente Roosevelt (ONU, 2014).

8 En el enfoque de la "modernización" se entendía por desarrollo el proceso que debería emprenderse en América Latina, África, Asia y Oceanía para sentar las bases que permitiesen reproducir las condiciones que caracterizaban a las naciones económicamente más avanzadas del mundo, como la industrialización, la alta tasa de urbanización y de educación, la tecnificación de la agricultura y la adopción generalizada de los valores y principios de la modernidad, incluyendo formas concretas de orden, racionalidad y actitud individual (Valcárcel, 2006). 
Asimismo, se hizo evidente una serie de efectos sociales de esta "modernización": migración del campo a la ciudad, urbanización deficiente, incremento de la pobreza urbana.

En este contexto de evidencias y reflexiones, el economista británico Dudley Seers (Seers, 1980) debatió la idea de que se pudiera hablar de un mejor desarrollo simplemente porque hubiera un crecimiento en la renta per cápita, sin que se dieran mejoras sensibles en otras dimensiones sociales como en los índices de pobreza, el empleo y la desigualdad. La redistribución de la riqueza surgió como un aspecto olvidado del crecimiento, que es necesario incorporar como objetivo para conseguir un verdadero desarrollo social. Desde esta óptica, se comenzó a considerar la satisfacción de las necesidades básicas de las personas como una de las prioridades del desarrollo, de manera que si los indicadores de las mismas no mejoraban, no podía afirmarse que se había avanzado en la consecución del desarrollo. Bajo este modelo, la prioridad fue satisfacer las necesidades básicas de los asentamientos humanos, particularmente de los más pobres (Cárdenas, 1998). El propulsor de este modelo, el economista Paul Streeten, señaló: "el objetivo de los esfuerzos a favor del desarrollo es proporcionar a todos los seres humanos la oportunidad de vivir una vida plena $[\ldots]$ la incumbencia fundamental del desarrollo son los seres humanos y sus necesidades" (Streeten, et al., 1986: 31).

Por otro lado, aunque desde finales de los años setenta y en los años ochenta comenzaron a discutirse las problemáticas ambientales, no fue sino hasta la década de los noventa cuando dicha temática logró permear en las agendas políticas. Fue así que paulatinamente fueron apareciendo las aproximaciones medioambientales en torno al concepto y la política del desarrollo. Algunos de estos enfoques fueron el "eco desarrollo", el "otro desarrollo", el "desarrollo sostenido" y el "desarrollo sustentable". El "eco desarrollo" buscaba armonizar cinco dimensiones: pertinencia social y equidad de las soluciones, prudencia ecológica, eficacia económica y dimensión cultural y territorial (Valcárcel, 2006). El "otro desarrollo" surgió con la creciente desilusión con el enfoque de la "modernización" y la inconformidad de la población con la "sociedad de consumo". La noción de "desarrollo sustentable", precedida por la noción de "desarrollo sostenido", apareció, por su parte, tras la exacerbación del deterioro del medio ambiente. Así, en el documento Estrategia mundial para la conservación que publicó la Unión Internacional para la Conservación de la Naturaleza y el World Wildlife Fund, se hace referencia a la necesidad de preservar los recursos vivos para un desarrollo sostenido, entendiéndose por éste "el crecimiento económico que no vulnera los ecosistemas" y que tiene un carácter permanente y de largo alcance (Valcárcel, 2006). Desde todas estas perspectivas, la variable del medio ambiente y la preservación de los recursos naturales son vistos como esenciales en el desarrollo, sin los cuales, éste es inconcebible.

Aunque estas nuevas nociones de desarrollo de aproximación ambientalista no son semejantes, sí tienen muchos atributos comunes, pues abordan de forma simultánea los desafíos que, en el contexto de su aparición, enfrentaba la sociedad: 
la pobreza, la destrucción del medio ambiente y el crecimiento económico; cuestionan, también, a la sociedad de consumo y la malversación de recursos que la acompañan; y enfatizan en la importancia de satisfacer las necesidades de toda la población, erradicando la pobreza y buscando la equidad.

Al conjunto de nociones ambientales del concepto de desarrollo se le agregan en el debate académico y político la noción basada en el "desarrollo humano". Bajo esta perspectiva se pretende una visión de desarrollo que coloque al ser humano y a los intereses colectivos de las mayorías como punto central, convergiendo a la posibilidad de potencialización de las capacidades de todos los individuos. Como se puede percibir, el concepto de desarrollo atenuó su carácter estrictamente económico y se transformó en un concepto más integral y, por consiguiente, más complejo, multidimensional e intangible.

Este planteamiento es compartido por Javier Elguea, quien sostiene que las ideas modernas sobre el progreso, crecimiento o desarrollo, se encaminan hacia el mejoramiento de la condición humana, de tal forma que el desarrollo material es un medio para la realización de las personas (Elguea, en Porras, 2011).

Amartya Sen, por su parte, ha ofrecido luz sobre la interrogante de cómo concebir el desarrollo desde una perspectiva más amplia, en donde la pobreza no se mide sólo por la falta de riqueza o de ingresos, sino en términos de "capacidades básicas insuficientes"; en este sentido, Sen apuntó que el desarrollo no debe de reducirse al aumento de la oferta de mercancías, sino que debe de acrecentar las capacidades de la gente. Para Sen, el proceso de desarrollo se ve cuando las personas tienen la capacidad de seleccionar entre varias opciones que les dan bienestar (Sen, 2004); es decir, el objetivo del desarrollo no es incrementar la riqueza, sino propiciar que la gente tenga dominio sobre su propia vida y disponga de un abanico amplio de opciones para, por ejemplo, tener acceso a servicios médicos que le permitan evitar enfermedades y vivir más años (Griffin, 2001). Las capacidades que señala Sen en el proceso de desarrollo están intrínsecamente relacionadas a la libertad del ser humano para lograr "una vida plena".

En la misma línea, el Programa de las Naciones Unidas para el Desarrollo (PNUD) ha elaborado lo que denomina Índice de Desarrollo Humano (IDH). Este indicador integra la dimensión del acceso a los recursos que el PIB per cápita puede representar, con registros que miden otras manifestaciones del desarrollo humano, estudiando tres variables: la salud, la educación y el ingreso. En otras palabras: a) la capacidad básica de contar con una vida larga y saludable, calculada a través de la esperanza de vida al nacer y de los índices de mortalidad infantil; b) el logro educativo de la población de un país estimado a través de los años promedio de escolaridad para personas mayores de 24 años, así como de los años esperados de escolaridad para personas entre seis y 24 años; y c) el acceso a los recursos que permiten gozar de un nivel de vida digno, el cual se calcula a partir del Ingreso Nacional Bruto (INB) (PNUD, 2014).

Con lo anterior podemos concluir que las diferentes nociones de "desarrollo" - la 
de "necesidades básicas", de Streeten; la de "las capacidades", de Sen: y la de "desarrollo humano", del Programa de las Naciones Unidas para el Desarrollo - conciben a los seres humanos como objetivo primordial del desarrollo mismo; esto comprende desde sus necesidades básicas, como la alimentación y la vivienda, hasta otras que involucran el acceso a servicios públicos, como el transporte, el empleo, la educación y la salud. En todas ellas, el desarrollo excede en mucho a lo meramente económico e incluye dimensiones sociales, políticas y culturales. El planteamiento es que las actividades económicas deben orientarse para garantizar la calidad de vida, en donde la participación y la expansión de las libertades humanas toman un rol central.

Es fácil distinguir cómo el concepto de desarrollo, sobre todo en las últimas décadas, ha evolucionado de manera acelerada de acuerdo al pensamiento y a los valores dominantes en la sociedad. El sustantivo "desarrollo" ha transitado de un asunto económico a un asunto de bienestar social $\mathrm{y}$ de desarrollo humano. Al estudiar estos aspectos es cuando surgen preguntas a las que es necesario buscar respuestas: ¿los modelos de desarrollo, cuyo objetivo principal es el bienestar del ser humano, reflejan la diversidad de género y sexual de los seres humanos? ¿Cuál es el subtexto de género y sexo implícito en ese concepto de ser humano? ¿Cuál es la concepción sobre la manera en que el tema de las diferencias de género y sexuales, así como las dinámicas de poder y distinción social a la que dan lugar en las sociedades, deben ser abordadas en la concepción del desarrollo e incluidas en las políticas de desarrollo globales, nacionales y regionales?

\section{MUJERES, GÉNERO Y DESARROLLO}

En 1970 la economista danesa Ester Boserup (2011) publicó el libro Woman 's Role in Economic Development en elquecriticalanocióndedesarrollo y las políticas de desarrollo entonces vigentes, por excluir a las mujeres. Según la investigadora, las políticas de desarrollo elaboradas desde 1945 han visto a las mujeres como sujetos pasivos del desarrollo y han considerado sólo a los hombres en los procesos de entrenamiento, transferencia tecnológica y financiera. Asimismo, denuncia que estos programas han estado centrados en la unidad doméstica, como unidad privilegiada estandarizada, y en los hombres, vistos bajo la noción de ser ellos los "proveedores" del hogar. El problema de esta perspectiva, señaló Boserup, es que no sólo desconoce el papel que las mujeres tienen en la producción agrícola y ganadera $\mathrm{y}$, en general, en la producción de alimentos, bienes y servicios, sino también desconoce que la unidad doméstica es un espacio de relaciones de poder, por lo que la ayuda para el desarrollo destinada a los hombres como sujetos principales de estas políticas, no necesariamente se traduce en un mayor bienestar para las mujeres o para los "dependientes", o no, en todo caso, de manera equitativa.

Esta poderosa crítica de Boserup logró colocarse en la Conferencia Mundial de la Mujer celebrada en la ciudad de México en 1975 y en la agenda de la "Década de la Mujer", establecida por la Organización de las Naciones Unidas ese mismo año. A partir de entonces, se institucionalizó la perspectiva que pretende hacer efectiva la inclusión de la mujer en el 
desarrollo, así como el reconocimiento a la importancia del trabajo productivo y reproductivo de las mujeres en las economías nacionales (Safa, 1995). También desde entonces y durante los años ochenta del siglo $\mathrm{XX}$, se multiplicaron las organizaciones no gubernamentales (ONG) que buscaban acceder a recursos financieros para el desarrollo con proyectos que incluían a las mujeres. Incluso se comenzó a considerar que las mujeres, en virtud del papel social y culturalmente asignado de "cuidadoras", eran más responsables, más ahorradoras, mejores administradoras y más justas en la aplicación de los recursos destinados al desarrollo en beneficio de sus hijas e hijos y de la comunidad (Jackson, 1994). Fue así que surgieron muchos programas de microcrédito destinados a grupos de mujeres en diversos países y regiones.

Cabe mencionar que la perspectiva de "las Mujeres en el Desarrollo" no fue la única. En esos mismos años surgió la perspectiva de "las Mujeres y el Desarrollo", una serie de reflexiones teóricas provenientes del marxismo que ponía énfasis en dos cosas: 1) en que el modelo de "las Mujeres en el Desarrollo" no cuestionaba el modelo económico neoliberal y 2) en que no reconocía que las mujeres siempre habían estado presentes en el desarrollo a través de su trabajo productivo y reproductivo. La perspectiva de "las Mujeres y el Desarrollo" echó mano de "la Teoría de la Dependencia", que cuestionó el modelo desarrollista de entonces, para plantear que las mujeres - y su trabajo doméstico no pagadohan sido fundamentales en el funcionamiento de sus sociedades. Asimismo, expuso que esta integración subordinada de las mujeres ha contribuido a sostener un orden económico internacional desigual entre el Norte desarrollado y el Sur explotado (Aguinaga et al., 2013).

Ambas perspectivas, sin embargo, serán cuestionadas por un planteamiento teórico feminista atento a la construcción social de las mujeres, en tanto sujetos genéricos y en tanto la manera en que sus identidades, prácticas y vínculos, se producen en relación con otras distinciones sociales como la clase, la etnia/ raza, el origen urbano/rural, la edad y el origen nacional. El cuestionamiento parte del análisis de la reproducción de las condiciones de vida de las mujeres y está sustentado en la idea de que sólo el cambio económico trae consigo el empoderamiento de las mujeres y un mayor bienestar. Esta perspectiva, llamada "Género y Desarrollo", propone ir más allá de los microcréditos para superar la pobreza y plantea la necesidad de atender la dominación y la violencia que sufren las mujeres (a menudo de parte de sus parejas hombres, o de otros hombres de la familia, y de la sociedad), la doble jornada laboral, el peso adicional de responsabilidad que los programas de combate a la pobreza y de desarrollo colocan en la vida de las mujeres, en su energía y en su bienestar (ahora a cargo no sólo de la reproducción y a menudo del trabajo productivo, sino también de realizar dentro del hogar la "agenda del desarrollo"), sus condiciones de educación y salud, y especialmente los retos en materia de salud reproductiva y salud sexual.

La perspectiva del "Género en el Desarrollo", a diferencia de la perspectiva del "Género y Desarrollo", pone un énfasis especial en 
la necesidad de transformar el sistema de género en su totalidad, con miras a construir relaciones más equitativas entre hombres y mujeres en todo el espectro de las relaciones y prácticas sociales, $\mathrm{y}$ en hacer de esto un objetivo y una estrategia de desarrollo. Por lo mismo, llama la atención sobre la necesidad de no hacer de la unidad doméstica la única unidad de análisis y de atención de las políticas de desarrollo, sino atender a otras instancias sociales de reproducción de la desigualdad y la inequidad de género (como la educación, la salud, el mundo laboral, el acceso a la justicia), así como asuntos colectivos relacionados con el desarrollo de infraestructuras públicas, la redistribución del ingreso, el acceso a la propiedad y la lucha contra otras formas de inequidad que afectan la vida de las mujeres, como la clase, la etnia/raza y la edad, por mencionar las más comunes.

Una plataforma inicial en donde se articuló en la agenda del desarrollo esta perspectiva de género, fue la CIPyD, celebrada en El Cairo, en 1994. En esta conferencia se estableció claramente una visión de crecimiento económico en el marco de un desarrollo sostenible que habilite a la mujer a fin de "garantizar que participe plenamente a todos los niveles en la vida social, económica y política de su comunidad" (ONU, 1994: 7). Dicha agenda fue ratificada y ampliada en la IV Conferencia Mundial sobre las Mujeres, celebrada en 1995 en Pekín, China (De la Cruz, 2007). Otro hecho relevante fue la Cumbre del Milenio, ${ }^{9}$ celebrada en 2000 , con la participación

9 En septiembre de 2000, los dirigentes del mundo se reunieron en la sede de las Naciones Unidas, en Nueva York, para aprobar la Declaración del Milenio. La Declaración, firmada por 189 Estados miembros de las Naciones Unidas, de 189 países, en donde se pactó encaminar esfuerzos para cumplir, antes del 2015, con ocho objetivos para mejorar las condiciones de vida de todas y todos en el mundo. El tercero de los llamados "Objetivos de Desarrollo del Milenio" refiere puntualmente la importancia de "promover la igualdad entre los géneros y la autonomía de la mujer". La igualdad de género implica que todos los hombres y mujeres tengan las mismas condiciones y oportunidades para ejercer de forma plena sus derechos humanos, sin importar su sexo ni identidad de género. Una igualdad que debe de ser procurada en todos los demás objetivos planteados en la cumbre, como señaló el propio Secretario General de Naciones Unidas, Ban Ki Moon: "La igualdad de género no es sólo un fin en sí mismo, sino que es un prerrequisito para alcanzar todos los objetivos internacionales del desarrollo, incluyendo las Metas del Milenio" (CINU, 2014). Esta visión sustenta e impulsa lo que se ha dado en llamar en los últimos años "la transversalización de la perspectiva de género" en la política pública de desarrollo.

El desarrollo centrado en género o "con perspectiva de género" significa que a mujeres y hombres les sean dadas iguales oportunidades para sobrevivir y crecer, así como contribuir y

se tradujo posteriormente en un mapa de ruta estableciendo objetivos a ser alcanzados para 2015. Los ocho "Objetivos de Desarrollo del Milenio" se basan en acuerdos adoptados en la década de los noventa, en conferencias y cumbres de las Naciones Unidas, y representan un compromiso de todas las naciones para reducir la pobreza y el hambre, disminuir las enfermedades, la inequidad entre los sexos, enfrentar la falta de educación, la falta de acceso a agua y saneamiento y detener la degradación ambiental. Para mayor información, puede remitirse al sitio web de la Comisión Económica para América Latina y el Caribe (CEPAL, 2014). 
beneficiarse de los procesos sociales, económicos y políticos. También significa que con la equidad como valor, las mujeres y los hombres deben disfrutar plenamente de los mismos derechos y deben tener acceso y control de los recursos que les permitan participar en la construcción de sociedades más democráticas, equitativas, seguras y sustentables.

\section{LA REPRODUCCIÓN Y LA SEXUALIDAD EN LA CONCEPCIÓN DEL DESARROLLO}

El feminismo nace en la modernidad para cuestionar el carácter sexista de sus supuestos culturales (como la asociación de la razón con la masculinidad) y efectos sociales, políticos y económicos para las mujeres $\mathrm{y}$, con ello, de su exclusión como participantes y beneficiarias activas de sus realizaciones y promesas de bienestar. Bajo este cuestionamiento, los temas relacionados con el papel de las mujeres en la reproducción biológica y social de las personas y de las sociedades siempre tuvieron un lugar relevante (Tong, 1989). Sin embargo, es hasta la reflexión de Boserup (2011) que los temas relacionados con la reproducción, como las mujeres y su posición en la familia, las dinámicas de poder dentro de la unidad doméstica o la importancia de su trabajo reproductivo y productivo no remunerado, adquirieron visibilidad en la concepción sobre el desarrollo. En las décadas siguientes, la reflexión y la visibilidad sobre estos temas se ampliaron y se profundizaron en los distintos países, lo cual tuvo un impacto claro en documentos internacionales sobre el desarrollo, como el Programa de Acción derivado de la CIPyD, celebrada en El Cairo en 1994. Como trataremos de mostrar en los siguientes párrafos, este evento abrió las puertas, como nunca antes, al tema de la reproducción y la sexualidad en la agenda del desarrollo.

El Programa de Acción CIPyD planteó una noción de desarrollo que articuló preocupaciones sobre la población, la pobreza, el crecimiento económico sostenido, las modalidades de producción y consumo, y el medio ambiente. La conferencia incorpora tres dimensiones generales al concepto de desarrollo: 1) la noción de un desarrollo socialmente equitativo y ecológicamente racional (ONU, 1994: 5); 2) la noción de que el derecho al desarrollo es parte integrante de los derechos humanos fundamentales; y 3) que la persona humana, y su potencial, es el sujeto central del desarrollo (ONU, 1994: 10).

El Programa de Acción de la CIPyD involucra una reflexión a favor de la necesidad de: 1) incorporar las políticas demográficas en la concepción del desarrollo; 2) visibilizar los nexos entre población, crecimiento económico sostenido y pobreza; y 3 ) visibilizar las relaciones entre población y medio ambiente. Es en este marco de preocupaciones que los temas de equidad de género, reproducción y sexualidad aparecieron de manera destacada en la discusión sobre el desarrollo. El argumento es claro: el tema poblacional (su crecimiento y distribución) es central en el desarrollo - $\mathrm{y}$ viceversa-, y sólo puede atenderse plenamente incorporando asuntos fundamentales como la habilitación (empoderamiento) de las mujeres en todos los aspectos de la vida social, de manera especial, en su capacidad para decidir sobre su reproducción. 
Para que eso ocurra, se dice, es necesario atender problemas como la desigualdad, la exclusión y la violencia que sufren dentro y fuera del hogar, así como el acceso a servicios integrales de salud y educación sexual y reproductiva. Se trata de un enfoque sobre el fenómeno de la población y el desarrollo que involucra como objetivos y metas: "el crecimiento económico sostenido en el marco del desarrollo sostenible; la educación, sobre todo de las niñas; la igualdad entre los sexos; la reducción de la mortalidad neonatal, infantil y materna; y el acceso universal a servicios de salud reproductiva, en particular de planificación de la familia y de salud sexual" (ONU, 1994: 8).

Dos conceptos se destacan en esta novedosa inclusión del tema de la reproducción (como parte del tema de la población, la equidad y la habilitación de las mujeres) en la concepción del desarrollo: la salud reproductiva y los derechos reproductivos. La salud reproductiva es definida como: "un estado general de bienestar físico, mental y social, y no de mera ausencia de enfermedades o dolencias, en todos los aspectos relacionados con el sistema reproductivo y sus funciones y procesos" (ONU, 1994: 37). La salud reproductiva entraña, pues, dos ámbitos importantes según esta definición:

La capacidad de disfrutar de una vida sexual satisfactoria y sin riesgos de procrear, y la libertad para decidir hacerlo o no hacerlo, cuándo y con qué frecuencia. Ésta última condición lleva implícito el derecho del hombre y la mujer a obtener información y de planificación de la familia de su elección, así como a otros métodos para la regulación de la fecundidad que no estén legalmente prohibidos, y acceso a métodos seguros, eficaces, asequibles y aceptables, el derecho a recibir servicios adecuados de atención de la salud que permitan los embarazos y los partos sin riesgos y den a las parejas las máximas posibilidades de tener hijos sanos (ONU, 1994: 37).

Por su parte, de los derechos reproductivos se dice:

\begin{abstract}
Abarcan ciertos derechos humanos [...]. Esos derechos se basan en el reconocimiento del derecho básico de todas las parejas e individuos a decir [sic] libre y responsablemente el número de hijos, el espaciamiento de los nacimientos y el intervalo entre éstos y a disponer de la información y de los medios para ellos y el derecho a alcanzar el nivel más elevado de salud sexual y reproductiva. También incluye su derecho a adoptar decisiones relativas a la reproducción sin sufrir discriminación, coacciones ni violencia, de conformidad con lo establecido en los documentos sobre derechos humanos (ONU, 1994: 37).
\end{abstract}

Cabe mencionar que estas definiciones no agotan todo el campo de la reproducción y de la agenda delineada en el Programa de Acción de la CIPyD, dado que a lo largo del documento es posible encontrar una diversidad de aspectos relacionados con la igualdad y equidad de género, con los temas de violencia, de integridad corporal, de acceso a educación, de necesidades de investigación de estos vínculos y de asuntos que conciernen a esferas no sólo de la reproducción biológica, sino social de los hijos e hijas y a las dinámicas de pareja. Esto último involucra dos cosas: 1) la propuesta de que de los varones, niños, adolescentes y adultos tengan también acceso a información, asesoramiento y servicios de salud reproductiva, a métodos 
de contracepción masculina voluntarios y adecuados, y a métodos encaminados a prevenir enfermedades de transmisión sexual, incluido el sida; y 2) la educación e incorporación de los hombres en la repartición por igual de las labores domésticas y de crianza de los hijos, de la responsabilidad de la planificación familiar y de la prevención de las enfermedades de transmisión sexual (ONU, 1994: 39).

Este último apartado apunta a la incorporación de los hombres y la masculinidad en el desarrollo, esto es, a la necesidad de pensar en los varones como sujetos que participan con sus ideologías, identidades y relaciones de género en las dinámicas de población y desarrollo y, en esa medida, inciden en ellas y son condicionados por ellas. Es importante señalar que la inclusión de este tema en el Programa de Acción de la CIPyD impulsó de manera significativa, en la década de los noventa y en la primera década del siglo XX, a los estudios de género de los varones, que ya venían desarrollándose dos décadas atrás (Núñez, 2009), no sólo en los temas de la salud sexual y reproductiva, sino en muchos otros ámbitos de la vida de pareja, familiar y social, como parte del conocimiento necesario para un desarrollo realmente incluyente y centrado en las personas.

Si bien, nos resulta imposible extendernos en este artículo sobre la diversidad de aspectos que se colocan en la mesa de discusión sobre el tema del género, la reproducción y el desarrollo, baste decir que su inclusión debería de movernos a revisar nociones de desarrollo que siguen limitándose a aspectos económicos, ambientales o de combate a la pobreza, y que no incluyen la equidad de género de manera central. Y así debería de ser, no sólo porque el género tiene que ver con la pobreza y su combate, con el crecimiento económico y el medio ambiente, sino porque la equidad y el bienestar de las personas debe de ser el objetivo central del desarrollo.

En este marco conceptual, nos interesa analizar el tratamiento de un tema poco abordado en la discusión sobre el desarrollo: la sexualidad.

El Programa de Acción de la CIPyD es relevante en lo que concierne a este tema porque inaugura, en un documento oficial de la ONU, la inclusión de la sexualidad en la agenda del desarrollo.Elabordajedelasexualidad,noobstante, está mediado por la incorporación del tema de la reproducción como parte de la preocupación general sobre la población. La cuestión de la reproducción, por su parte, es abordada desde las nociones de derechos reproductivos y salud reproductiva. Así, la sexualidad aparece, en primera instancia, en su relación con la salud y la reproducción (en su "definición amplia" que, se nos hace saber, "incluye la planificación de la familia y la salud sexual").

A lo largo del documento se menciona una serie de problemas importantes que hay que eliminar, prevenir o atender pues afectan la salud sexual y reproductiva y, por tanto, el desarrollo: la pandemia del VIH-sida, las ITS (Infecciones de Transmisión Sexual), la violencia sexual, la violación y el incesto, la mutación genital, la pornografía, la prostitución y el abuso sexual, (ONU, 1994: 31). Las personas afectadas somos todos en distintos grados: menores, adolescentes, jóvenes y adultos, hombres y mujeres, se señala. 
Aunque la sexualidad aparece mencionada en el documento en relación con la salud y la reproducción, el Programa de Acción de la CIPyD dibuja otras posibilidades de comprensión del vínculo sexualidad-desarrollo. Así, en una de las definiciones de la salud reproductiva, se dice: "la salud reproductiva entraña la capacidad de disfrutar de una vida sexual satisfactoria y sin riesgos de procrear" (ONU, 1994: 37). En otra definición, se dice que el objetivo de la salud sexual: "es el desarrollo de la vida y de las relaciones personales y no meramente el asesoramiento y la atención en materia de reproducción y de enfermedades de transmisión sexual" (ONU, 1994: 37). Así, la sexualidad se incluye en la noción del desarrollo no sólo por su valor para conseguir una buena salud reproductiva, sino también porque se reconoce la importancia de una vida sexual satisfactoria en los individuos y de su papel en el desarrollo de las relaciones interpersonales.

Un año más tarde, aunque de forma limitada, la Plataforma de Acción de Pekín de 1995 continuará esta línea de discusión de El Cairo, al tratar los derechos humanos de las mujeres en relación con la sexualidad, en su Párrafo 96. Sin embargo, y a pesar de que la perspectiva de los derechos humanos con relación a la sexualidad abre el camino al reconocimiento de los "derechos sexuales" como tales, la enunciación de los mismos no parece aportar ninguna novedad a lo dicho en El Cairo: tener control y decidir libremente en asuntos relacionados con su sexualidad, incluyendo su salud sexual y reproductiva, libre de coerción, discriminación y violencia; igualdad de hombres y mujeres en los asuntos de las relaciones sexuales y la reproducción, incluyendo el respeto integral de la persona; respeto mutuo, consentimiento y responsabilidad compartida del comportamiento sexual y sus consecuencias. Como lo señala la activista y estudiosa Sonia Correa (2008), el Párrafo 96 se quedó muy por debajo de la promesa implícita en la noción de derechos sexuales, pues no incluyó asuntos como la orientación sexual, la situación de las personas transexuales, transgéneros e intersexuales, o la violencia homofóbica. A decir de la autora, la presión de los grupos y países conservadores (como la Conferencia de los Países Islámicos) impidieron el avance en esos temas en el evento y en reuniones posteriores, como en la revisión quinquenal de 2000, y otros subsiguientes de la ONU (Correa, 2008: 28).

El trabajo de organizaciones civiles globales, sin embargo, ha sido fundamental en la construcción de una visión más rica e inclusiva de los derechos sexuales; es el caso de la Asociación Mundial para la Salud Sexual que, en el Congreso Mundial de Sexología celebrado en Hong Kong en agosto de 1999, estableció con claridad que los derechos sexuales son un componente fundamental de los derechos humanos universales y que se basan en la libertad, dignidad e igualdad inherentes a todos los seres humanos.

Así, en la medida en que la salud es un derecho humano, también lo es la salud sexual, que solo puede ser conseguida con el reconocimiento, la promoción, el respeto y la defensa de los derechos sexuales, por todas las sociedades y por todos los medios. La Declaración de Derechos Sexuales es importante porque va más allá de los 
asuntos reproductivos o de violencia y coerción tal y como hasta entonces habían sido definidos, e incluyen, por primera vez, el derecho a la igualdad sexual y a no ser discriminado en virtud del sexo, el género o la orientación sexual. ${ }^{10}$

Este planteamiento de los Derechos Sexuales finalmente se integrará y ampliará, en su concepción y alcance, gracias a la movilización de la sociedad civil internacional en el ámbito de la ONU en varios documentos importantes: la Resolución de Brasil sobre Derechos Humanos y Orientación Sexual que fue presentada al Consejo Económico y Social de las Naciones Unidas en el año 2003; las definiciones de Sexualidad, Salud Sexual y de Derechos Sexuales propuestas por la Organización Mundial de la Salud (OMS) en el año $2006^{11}$ y los "Principios de Yokarta: Principios sobre la aplicación de la legislación internacional de derechos humanos en relación con la orientación sexual y la identidad de género".

La presentación de los Principios de Yokarta tuvo lugar el 26 de marzo de 2007 en el Consejo de Derechos Humanos de la ONU, en Ginebra, y fue ratificada posteriormente por la Comisión

10 Véase la Declaración de los Derechos Sexuales en World Association for Sexual Health (2014).

11 La definición de sexualidad de la OMS es la siguiente: "La sexualidad es un aspecto central del ser humano, presente a lo largo de su vida. Abarca al sexo, las identidades y los papeles de género, el erotismo, el placer, la intimidad, la reproducción y la orientación sexual. Se vivencia y se expresa a través de pensamientos, fantasías, deseos, creencias, actitudes, valores, conductas, prácticas, papeles y relaciones interpersonales. La sexualidad puede incluir todas estas dimensiones, no obstante, no todas ellas se vivencian o se expresan siempre. La sexualidad está influida por la interacción de factores biológicos, psicológicos, sociales, económicos, políticos, culturales, éticos, legales, históricos, religiosos y espirituales" (WHO, 2006: 5).
Internacional de Juristas. El documento establece con claridad, en un organismo internacional de la ONU, un marco de derechos humanos reconocidos internacionalmente para la población lésbica, gay, bisexual y transgénero/transexual, en el mundo.

Estos avances en el marco de los documentos internacionales y de las sociedades y gobiernos de muchos países del mundo, han incidido en algunas agencias gubernamentales y organismos civiles globales que reflexionan sobre el vínculo entre sexualidad y desarrollo; dos llaman en particular nuestra atención: el presentado por la Agencia de Desarrollo Internacional Sueca (ADIS, por sus siglas en español), titulado "Sexuality: A Missing Dimension in Development" (elaborado por Anne Runeborg) y el "Índice de Inclusión Social 2014", de la Americas Quarterly. The Policy Journal for our Hemisphere (elaborado por Rebecca Bintrim, Wilda Escarfuller, Christopher Sabatini, Alana Tummino y Adam Wolsky).

La ADIS busca, con su política global para el desarrollo, el mejoramiento de la calidad de vida de las personas a partir de dos perspectivas: la de la gente que vive en pobreza y la del enfoque basado en derechos. Desde la perspectiva de los derechos, señala esta agencia: "la sexualidad importa porque tiene que ver con el poder y sin los derechos básicos sobre nuestros propios cuerpos y sobre elecciones de vida fundamentales [cuándo y con quién casarse o entrar en una relación de pareja, cuándo y con quién tener hijos, por ejemplo], muchos otros derechos no son alcanzables. La sexualidad es importante porque los derechos sexuales son los derechos de todos" (Runeborg, 2008: 8). Con base en este planteamiento, el 
documento proclama "la necesidad de una política sexual basada en derechos en todos los países". Con relación a la segunda perspectiva, señala: "la carencia de derechos sexuales es en sí misma una dimensión de la pobreza, que a su vez produce un conjunto de resultados relacionados con la pobreza, desde la exclusión social y la inseguridad física a una mayor vulnerabilidad a las enfermedades, el hambre y la muerte" (Runeborg 2008: 8). Con esta perspectiva, el documento de la ADIS muestra cómo la sexualidad, en su diversidad y complejidad, se conecta con asuntos de desarrollo fundamentales, también de manera rica y compleja: la pobreza; los derechos humanos, la democracia y la participación política; la igualdad de género y el empoderamiento de la mujer y su papel en el desarrollo; la epidemia del VIH, la seguridad y la violencia de género ( $\mathrm{y}$ todo esto con la economía); el medio ambiente, el clima y los desastres naturales; los servicios de salud y la educación; la infraestructura; la agricultura; y la migración. Algo importante a destacar en el documento de la ADIS es la incorporación de la población "lésbica, gay, bisexual, transgénero/ transexual e intersexual (o la que es vista como tal)" en la problemática del desarrollo, en la medida en que cada una de ellas enfrenta una "amplia discriminación y persecución"

Con relación al Índice de Inclusión 2014, destaca entre sus "claves para variables y fuentes" (que incluyen el crecimiento del PIB, el porcentaje del PIB invertido en programas sociales, los derechos políticos, el porcentaje de la población que vive con más de cuatro dólares al día, la inscripción en la escuela secundaria, el empoderamiento personal, la inclusión financiera, el porcentaje de acceso a empleo formal, la capacidad de respuesta del gobierno, el acceso a vivienda adecuada, la participación ciudadana, los derechos civiles, los derechos de la mujer) "los derechos LGBT", basados en "el Índice de Simpatía hacia los Homosexuales (GayFriendliness Index) de Javier Corrales [2009], cuyo puntaje refleja la existencia y permisibilidad de las relaciones entre personas del mismo sexo, las leyes vinculadas con la no discriminación y las leyes vinculadas con las relaciones entre personas del mismo sexo" (Bintrim, et al., 2014: 5). Lo importante de este documento es que apunta a una concepción del desarrollo que incluye no sólo a la sexualidad, sino una visión no heterosexista de la sexualidad.

\section{LA PERSPECTIVA TEÓRICA QUEER DE LA SEXUALIDAD Y EL DESARROLLO}

Cuando se analiza la manera en que se ha venido incorporando el tema de la sexualidad en la discusión sobre el desarrollo, se puede distinguir el tránsito de una visión subordinada a la noción de reproducción (salud reproductiva y derechos reproductivos), la equidad de género (entendida como relación de hombres y mujeres) y prevención de la violencia, a discusiones crecientes que incorporan una visión desde los derechos humanos e inclusiva de la diversidad sexo-genérica.

No obstante estos avances, creemos que se sigue careciendo de un planteamiento teóricoconceptual coherente sobre la sexualidad y el poder en las sociedades modernas, que sirva de base para sustentar una reflexión sistemática e integradora de la sexualidad en el desarrollo: 
una reflexión que incorpore las discusiones contemporáneas y la abundante evidencia sobre la sexualidad como un dispositivo de poder de la modernidad fuertemente vinculado a la administración de la población, como lo señala Michel Foucault (2012); como una construcción social e histórica, y no un simple impulso natural (Weeks, 1985); como un sistema ideológico que reproduce discursos dominantes sobre el cuerpo, el género, el erotismo, la reproducción y la familia presentes en la sociedad (Butler, 1990, 1991) y construye relaciones de poder complejos en los sujetos y en la sociedad toda; un dispositivo de poder por medio del cual se vuelven inteligibles y legítimas ciertas modalidades de existencia sexual y no otras (Butler, 1991; Foster 2000), y cuyo efecto es devastador en términos humanos: personas y colectivos sociales que sufren invisibilidad, exclusión, segregación, persecución, discriminación, subordinación, control, exterminio, así como un estado general de homofobia que alimenta ideologías, identidades y relaciones machistas.

Las derivaciones de una perspectiva de este tipo para pensar el desarrollo son múltiples y nos resulta imposible abordarlas aquí. No obstante, nos parece importante incorporar el planteo teórico queer (Jagose, 1996) para cuestionar tres ideologías del sistema sexo-género dominante que se hacen presentes en documentos como el Programa de Acción de la CIPyD y otros: 1) la noción binaria de los sexos; 2) la noción binaria de los géneros; y 3) el binarismo erótico y el heterosexismo (Núñez, 2011).

\section{El binarismo sexual}

El binarismo sexual es la ideología y práctica de construir dos sexos de los cuerpos humanos, a partir del supuesto binarismo cromosómico, gonadal y genital de la especie.

Esta es una posición equivocada pues en la especie humana no sólo existen personas con el par cromosómico XX y XY, sino personas con un trío cromosómico $\mathrm{XXY}, \mathrm{XYY}, \mathrm{XXX}$, personas con un solo cromosoma $\mathrm{X}$, o incluso con más variantes cromosómicas. Respecto a las gónadas, no sólo existen las personas con testículos o con ovarios, sino también las personas que tienen ambas gónadas, a quienes en Occidente se les dio el nombre, por muchos años, de "hermafroditas". Respecto a lo genital, también es difícil asentar el binarismo sexual con firmeza, pues no sólo existen seres humanos con pene y testículos o con vulva y vagina, sino quienes tienen una diversidad de arreglos de tejidos genitales. Se trata de una diversidad sexual (cromosómica, gonadal y genital) que abarca, según algunos cálculos, entre el 1\% y el 3\% de la población mundial (FaustoSterling, 1993).

En el plano del "sexo biológico", desde el activismo y desde ciertos planteos académicos, a esta diversidad se le ha dado en llamar "intersexualidad" (no sin discusiones y resistencias, como es de esperarse). Las personas intersexuales, cuyo movimiento ha emergido en las últimas décadas, demandan reconocimiento, trato digno, respeto a su integridad corporal y a sus decisiones sobre su cuerpo y sobre la identidad sexual que deseen asumir, así como el rechazo a las intervenciones quirúrgicas de reasignación sexual 
sin su pleno consentimiento (a través de la cirugía pediátrica), por considerarlas una agresión a su integridad corporal y, por lo tanto, a sus derechos humanos. Las personas intersexuales organizadas luchan por dejar de ser vistas como "fenómenos", "enfermos", "síndromes" o "anormalidades", para ser reconocidos plenamente como seres humanos dignos.

La intersexualidad plantea una realidad humana que exige su reconocimiento e inclusión a en el ámbito jurídico, social, cultural, económico y político, y la plena consideración de sus necesidades como seres humanos en los planes y programas de desarrollo regionales, nacionales y globales. La no inclusión social, hasta ahora, de esta población, ha afectado no sólo su salud, educación y capacidad económica y, con ellos, su inclusión misma como sujetos actores y beneficiarios del desarrollo, sino también su integridad corporal, su dignidad como seres humanos y su vida misma.

A las personas intersexuales no se les reconoce y no se les incluye en el desarrollo cuando, como en el caso del Programa de Acción de la CIPyD, se reproduce acríticamente la ideología que sólo acepta la existencia de dos cuerpos y de dos sexos en la especie humana. Como ejemplo, pueden citarse frases llenas de buenas intenciones, pero inadecuadas, como cuando se dice que se busca: "promover la equidad y la igualdad entre los sexos", o "la erradicación de todas las formas de discriminación entre los sexos" (ONU, 1994: 11), pero sólo se entiende por sexo al hombre y a la mujer, y por discriminación a la que ocurre contra las mujeres.

\section{El binarismo de género}

El binarismo de género es otro dispositivo de poder. Lo podemos definir como la ideología y práctica que se apoya en el binarismo sexual, para "naturalizar" el comportamiento y los papeles sociales asignados a hombres y mujeres (su masculinidad y femineidad).

La idea básica del binarismo de género es que del cuerpo/sexo macho se deriva naturalmente la masculinidad y del cuerpo/sexo hembra, la femineidad. La lucha feminista contra los estereotipos de género ha logrado incidir de manera importante en este dispositivo de poder; incluso ha logrado que se incorpore en la agenda de desarrollo global, a través de nociones como igualdad y equidad de género, la lucha contra los estereotipos de género, la habilitación o empoderamiento de género y la plena inclusión de las mujeres en la planeación y la aplicación de las políticas de desarrollo. Sin embargo, el impacto del binarismo de género no se agota y no se puede agotar en esta agenda, pues afecta a más personas que no están siendo incluidas.

El binarismo de género ignora a las personas que transgreden el género esperado en algún aspecto o a lo largo de su vida. Nos referimos aquí tanto a las personas transexuales, como a las personas transgénero y andróginas. La transexualidad alude a una realidad humana ampliamente documentada que refiere a la existencia de personas que tienen una disonancia entre su sexo biológico y su identidad de género. La sensación de vivir en un cuerpo equivocado, de sentirse hombres atrapados en un cuerpo de mujer, o viceversa, es la típica sensación descrita por una 
persona transexual. La invisibilidad, exclusión, segregación, persecución, control y subordinación económica, social, cultural y política de las personas transexuales condiciona fuertemente su situación de pobreza, sus altas tasas de morbilidad y mortalidad (una baja esperanza de vida) y sus muy bajos niveles de vida y de bienestar. Es el caso también de la transgeneridad, que autoras como Josefina Fernández (2004) describen como una realidad humana que se caracteriza por la transgresión del género esperado socialmente (el hombre "tiene que ser masculino y la mujer femenina") ya sea gestual, en la vestimenta, en el cuerpo (especialmente en sus rasgos sexuales secundarios: vello, senos, cadera, por ejemplo), en el comportamiento o en el nombre con el que desean ser referidas socialmente. La transgresión del género puede asumir modalidades $\mathrm{y}$ temporalidades diversas.

La condición transexual y transgénero en contextos sociales de ideologías de binarismo sexual y de género, es objeto, desde la infancia, de una diversidad de violencias, entre ellas, bullying, acoso, expulsión de los hogares, depresiones, suicidios e ideación suicida, agresiones, acoso policiaco, violaciones, negación de servicios de salud adecuados, discriminación cotidiana, negación legal, pena de muerte, homicidos. Se trata de una violencia estructural de las sociedades patriarcales que repercute en las condiciones de pobreza de los afectados. La inmensa mayoría de personas transexuales y transgénero sufren una violencia y exclusión económica que no sufren otras personas de su propia clase social en las sociedades donde habitan, dado que al ser expulsados de sus hogares no reciben herencias, no acceden a la tierra $u$ otras propiedades, no son objeto de atención de las políticas públicas de combate a la pobreza, no son sujetos de créditos ni de programas compensatorios que sí favorecen a las mujeres (aun cuando muchas de ellas se consideren a sí mismas mujeres). Asimismo, su exclusión o expulsión temprana de las escuelas, su falta de inclusión en las instituciones de salud o del empleo formal, agrava aún más su situación de pobreza.

Una concepción del desarrollo que no toma en cuenta a las personas transexuales y trasngénero, no es una concepción inclusiva y falla en la promesa de igualdad, equidad, respeto a los derechos humanos y búsqueda de bienestar y desarrollo humano.

\section{El binarismo erótico y el heterosexismo}

Un tercer dispositivo de poder en el sistema sexogénero, desde la teoría queer, es el binarismo erótico y el heterosexismo (Rich, 1993; Katz, 1990; Núñez, 2011).

Se trata de la ideología y práctica que sólo contempla dos preferencias sexuales (la heterosexualidad y la homosexualidad) y que las jerarquiza, considerando a la heterosexualidad como la única natural, normal o acorde a un plan divino. En este marco de concepciones y valores, las personas que sienten atracción y/o mantienen relaciones afectivas y eróticas con otras personas del mismo sexo o, de manera indistinta, a hombres, mujeres, intersexuales, transexuales, con todo y su diversidad de expresiones de género, también han sido y son objeto de persecución, extermino, 
segregación, exclusión, subordinación, opresión, control, reclusión y de violencias múltiples, físicas y verbales, en distintas sociedades humanas que afectan sus posibilidades de conseguir un estado integral de salud física, emocional, mental y social. La homofobia -incluímos aquí la lesbofobia, la bifobia, la transfobia y en general el rechazo a la disidencia sexo-genérica-, en tanto que ideología y práctica supremacista, es factor de exclusión en educación, empleo, salud y participación institucional, lo que dificulta el acceso a bienes y servicios, a propiedades, financiamientos, programas gubernamentales, desarrollo laboral y profesional, y que repercute en la pobreza de muchos sujetos o en cargas adicionales que exigen un sobreesfuerzo para compensar el rechazo social, en detrimento de su salud física, emocional, mental y social.

Nos equivocamos, sin embargo, si creemos que la homofobia, como parte central del dispositivo de poder del sistema sexo-género que construye el machismo como ideología y práctica de supremacía masculina, y como dispositivo de poder del sistema sexo-género (Núñez, 2011), sólo afecta a las personas consideradas homosexuales. Por el contrario, en la medida en que se integra en las políticas de género que participan en la construcción de las identidades de todos los hombres y las mujeres, la homofobia afecta a todas las personas (Kimmel, 1997).

La homofobia y la misoginia operan, principalmente, como amenaza en la infancia, adolescencia y adultez de muchos varones e instituye el miedo a ser o parecer "homosexual", "femenino", "como las mujeres", "poco hombre", etcétera. La homofobia, instalada en la psique masculina, afecta la calidad de vida de los hombres al afectar la calidad de sus vínculos de pareja, de amistad, de paternidad, de trabajo, pero también su salud, como lo demuestran los índices diferenciados de morbilidad y mortalidad de hombres y mujeres, según los cuales los hombres son más proclives a morir por accidentes automovilísticos, violencia homicida o por cirrosis (por alcoholización), así como por enfermedades prevenibles (De Keijzer, 1997). Las actitudes temerarias, las prácticas de riesgo, la competencia, el sentido de "honor masculino", la sensación de invulnerabilidad, como rasgos de personalidad masculinos esperados y transmitidos en sociedades patriarcales, se fincan en ideologías de género homofóbicas y machistas. Esto también afecta a mujeres, niños, niñas y otros varones, quienes a menudo sufren violencias múltiples a mano de otros hombres (Kaufman, 1987). El machismo también está presente en la organización subjetiva y en la identidad de las personas involucradas en el narcotráfico y en la delincuencia organizada, fenómenos sociales que amenazan las posibilidades de desarrollo de numerosos países como México.

La estimación del costo económico de la homofobia es un tema novedoso en el ámbito global, sin embargo, independientemente de su peso en las cuentas nacionales, si se quiere ser coherente con una noción de desarrollo centrado en la persona, en el ser humano, en sus características y capacidades, se tiene que atender y eliminar este dispositivo de poder heterosexista y homofóbico, así como elaborar un programa de acción que 
contemple la plena inclusión de las personas con su diversidad sexual de género y erótica, en las sociedades y en el concierto global, no solo como objetos de desarrollo, sino también como sujetos del mismo. Asimismo, se requiere entender que la lucha contra la homofobia y el integrismo sexual, de género y de orientación sexual, repercutiría en la construcción de subjetividades masculinas pacifistas, respetuosas del medio ambiente, más proclives al cuidado del otro, al respeto, la equidad y la solidaridad.

\section{REFLEXIONES FINALES}

El concepto de desarrollo hunde sus raíces en la promesa moderna de libertad y bienestar, una modernidad que en sus orígenes fue excluyente y tuvo un claro subtexto clasista, sexista, racista, homofóbico y profundamente dañino del medio ambiente. Bajo la presión de diversos colectivos sociales, la modernidad y el desarrollo mismo han sido analizados y sometidos a transformaciones diversas. Las concepciones y prácticas de desarrollo actuales involucran nociones no sólo de crecimiento económico y combate a la pobreza, sino de sostenibilidad ambiental, igualdad, equidad, inclusión y participación de amplios colectivos sociales. Temas como la equidad de género y la salud y los derechos reproductivos, reconocen la importancia de asuntos antes considerados "privados" en las dinámicas de población y desarrollo.

En este marco fue que, hace un par de décadas, se incluyó a la sexualidad en la agenda del desarrollo. Sin embargo, consideramos que esta inclusión inicial, subordinada a la discusión sobre reproducción y a preocupaciones de índole demográfica, debe de dar paso a una inclusión más completa y profunda, atenta a las dinámicas sociales y de poder en el ámbito de la sexualidad.

Esta tecnología de poder compleja que actúa sobre la vida sexual de la gente y dificulta la realización de los derechos humanos a muchas personas, debe ser analizada en relación con el concepto de desarrollo y con la agenda global, nacional y regional del desarrollo, no sólo porque excluye a sectores amplios de la población, condenándolos a la pobreza y a bajos índices de desarrollo humano, sino además porque los temas de la sexualidad, como aquí la hemos conceptualizado, repercuten en la pobreza social, a través de su impacto en los indicadores de morbilidad y mortalidad (accidentes, adicciones, cirrosis, VIH-sida, homicidios), en la violencia social y el deterioro de las instituciones (daño ambiental, narcotráfico, violencia patrimonial). Como señala la Agencia Internacional para el Desarrollo de Suecia, el hecho de que una sociedad no garantice los derechos sexuales, es en sí mismo un indicador de pobreza, pues vuelve incluso a los pobres, más pobres en derechos y en sus posibilidades de bienestar integral físico, emocional, mental y social, algo que a su parecer repercute en su pobreza.

Uno de los objetivos centrales de la inclusión de la sexualidad en la agenda del desarrollo, es eliminar las desigualdades basadas en la diversidad/disidencia sexo-genérica que limitan actualmente las posibilidades de los individuos para desarrollar y ejercer de forma plena sus capacidades, tanto en beneficio propio como para 
el bien de la sociedad en general, así como facilitar el acceso a los recursos para lograr un desarrollo centrado en la persona. Así, los organismos e instituciones internacionales relacionadas con las políticas de desarrollo, también deben empezar a ser analizados desde esta perspectiva, a fin de que sean inclusivos.

Finalmente, deseamos apuntar un tema adicional que esperamos poder desarrollar en un futuro artículo: la inclusión de la sexualidad en la concepción y en las políticas de desarrollo puede verse no sólo desde las consecuencias negativas producidas por la ausencia de las condiciones adecuadas para su ejercicio (como en el caso del embarazo adolescente, la violencia, el tráfico y la explotación sexual, o la epidemia del VIH-sida), sino como un área de oportunidad para individuos, comunidades, instituciones, regiones y países.

Un ambiente inclusivo, libre de homofobia y respetuoso de la diversidad sexual, de género y de orientación sexual, crearía nuevas posibilidades de migración de talentos, ambientes más cordiales de trabajo y convivencia, así como nuevas posibilidades de inversión en regiones y países. El desarrollo del turismo inclusivo (LGBTT), es un pequeño ejemplo de las áreas de oportunidad que se generan. Las comunidades que ofrecen ambientes de tolerancia, respeto e inclusión han visto así diversificadas sus fuentes de ingreso a través del turismo LGBTT.

Para concluir, creemos que el planteo teórico queer contribuye a fortalecer la conceptualización de la sexualidad y desarrollo, al tiempo que da un nuevo vigor a la agenda planteada con relación a este binomio. $\curvearrowright$ 


\section{BIBLIOGRAFÍA}

Aguinaga, Margarita, Miriam Lang, Dunia Mokrani y Alejandra Santillana (2013), "Development critiques and alternatives: a feminist perspective", en Miriam Lang y Dunia Mokrani (eds.), Beyond Development, consultado el 9 de julio de 2015, pp. 41-59, URL: http://www.rosalux.de/fileadmin/rls_uploads/ pdfs/sonst_publikationen/BeyondDevelopment.pdf

Bauman, Zygmunt (1992), Libertad. Madrid, Alianza.

Bintrim, Rebeca, Wilda Escarfuller, Christopher Sabatini, Alana Tummino y Adam Wolsky (2014), "The Social Inclusion Index 2014”, en Americas Quarterly. The Policy Journal for our Hemisphere (verano), consultado el 19 de junio de 2015, pp. 54-69, URL: http://www.americasquarterly.org/charticles/ socialinclusionindex2014/social_inclusion_index_2014.pdf

Boserup, Ester (2011), Woman's Role in Economic Development. London, New York, Earthscan.

Butler, Judith (1990), Gender Trouble. Feminism and the subversion of identity. New York, Routledge.

Butler, Judith (1991), "Imitation and gender insubordination", en Diana Fuss (ed.), Inside/Out. Lesbian theories, gay theories. Nueva York, Routledge.

Butler, Judith (1993), "Imitation and gender insubordination", en Henry Abelove, Michele Barale y David Halperin (eds.), The Lesbian and Gay Reader. New York, Routledge.

Cárdenas Jirón, Luz Alicia (1998), "Definición de un marco teórico para comprender el concepto de desarrollo sustentable", en Revistainvi, vol. 13, núm. 33, consultado el 12 de noviembre de 2014, pp. 3-20, URL: http://www.revistainvi.uchile.cl/index.php/INVI/article/view/228/990

CEPAL (2014), “Objetivos de desarrollo del Milenio en América Latina y el Caribe”, en Comisión Económica para América Latina y el Caribe, consultado el 19 de noviembre de 2014, URL: http://www.cepal.org/ mdg/docs/iadbpublicdoc.pdf

CINU (2014), "Ficha informativa sobre género y desarrollo No.1”, en Centro de Información de las Naciones Unidas, consultado el 19 de noviembre de 2014, URL: http://www.cinu.org.mx/gig/Documentos/genero.pdf 
Colmegna, Paula y Alejandro Isla (2007), “La importancia de la cultura y la política en los procesos de desarrollo", en Revista Mad, núm. 16, consultado el 19 de noviembre de 2014, pp. 93-107, URL: http://www.revistamad.uchile.cl/index.php/RMAD/article/viewFile/13949/14250

Corrales, Javier (2009), Amherst College. Obtenido de Amherst College: URL: https://www.amherst.edu/media/ view/95641/original/Corrales\%2BGay\%2BFriendliness\%2BIndex.pdf

Correa, Sonia (2008), “Cruzando la línea roja: cuestiones no resueltas en el debate sobre los derechos sexuales", en Ivonne Szasz y Guadalupe Salas (coords.), Sexualidad, derechos humanos y ciudadanía. Diálogos sobre un proyecto en construcción. México, El Colegio de México, pp. 25-55.

De Keijzer, Benno (1997), "El varón como factor de riesgo. Masculinidad, salud mental y salud reproductiva", en Esperanza Tuñón (coord.), Género y salud en el sureste de México. México, ECOSUR, UJAT.

De la Cruz, Carmen (2007), Género, derechos y desarrollo humano. San Salvador, Programa de Naciones Unidas para el Desarrollo.

De Lauretis, Teresa (1993), "Sexual Indifference and Lesbian Representation”, en Henry Abelove, Michele Barale y David Halperin (eds.), The Lesbian and Gay Reader. New York, Routledge.

Elias, Norbert (2009), El proceso de la civilización. Investigaciones sociogenéticas y psicogenéticas. México, Fondo de Cultura Económica.

Fausto-Sterling, Anne (1993), “The Five Sexes. Why male and female are not enough”, en The Sciences, consultado el 19 de noviembre de 2014, pp. 20-24, URL: http://www.uta.edu/english/timothyr/Fausto-Sterling.pdf

Fernández, Josefina (2004), Cuerpos desobedientes: travestismo e identidad de género. Buenos Aires, Edhasa.

Foster, David (2000), Producción cultural e identidades homoeróticas. Teoría y aplicaciones. San José, Editorial Universidad de Costa Rica.

Foucault, Michel (2012), Historia de la Sexualidad 1. La voluntad del saber. México, Siglo XXI.

Fuss, Diana (1991), Inside/Outside. Lesbian Theories, Gay Theories. New York, Routledge. 
González, Natividad, Liliana Bergesio y Laura Golovanevsky (2014), “La Feria binacional de Camélidos y las Instituciones del desarrollo", Antípoda, núm. 18, consultado el 17 de noviembre de 2014, pp.189-213, URL:http://antipoda.uniandes.edu.co/datos/pdf/descargar.php?f=./data/Revista_No_18/n18a09.pdf.

Griffin, Keith (2001), Desarrollo Humano: origen, evolución e impacto. Barcelona, Icaria.

Jackson, Cecile (1994), "Gender analysis of green development”, en Ted Benton y Michael Redclift (eds.), Social theory and the global environment. London, Routledge.

Jagose, Annamarie (1996), Queer theory: an introduction. New York, New York University Press.

Jameson, Frederic (1981), The Political Unconscious: Narrative as a Socially Simbolic Act. New York, Cornell University Press.

Katz, Jonathan Ned (1990), “The Invention of Heterosexuality”, en Socialist Review, vol. 20, núm. 1, pp. 7-34.

Kaufman, Michael (1987), "The construction of masculinity and the triad of men's violence”, en Michael Kaufman (coord.), Beyond Patriarchy: Essays by Men on Pleasure, Power, and Change. Toronto, Oxford University Press, pp. 1-29.

Kimmel, Michael (1997), "Homofobia, temor, vergüenza y silencio en la identidad masculina”, en Teresa Valdés y José Olavarría (eds.), Masculinidad/es: poder y crisis. Santiago, FLACSO Chile/ ISIS Internacional, pp. 49-62.

Lyotard, Jean Francois (1979), The Postmodern Condition: A Report on Knowledge. Minneapolis, University of Minnesota Press.

Núñez, Guillermo (1993), “La metanarrativa de progreso y la emergencia de subalternidades: el caso de la Sierra en Sonora, México”, en La revista de El Colegio de Sonora, núm. 6, pp.77-91.

Núñez, Guillermo (1999), Sexo entre varones, poder y resistencia en el campo sexual. México, Miguel Ángel Porrúa, PUEG/UNAM, El Colegio de Sonora.

Núñez, Guillermo (2009), "Reflexiones para una mesa de diálogo que apenas empieza: feminismos y 
estudios de género de los hombres en México", en GénEros, año. 16, núm. 6, pp. 35-53.

Núñez, Guillermo (2011), ¿Qué es la diversidad sexual? Reflexiones desde la academia y el movimiento ciudadano. Ecuador, CIAD/Universidad Politécnica Salesiana.

ONU (1994), Informe de la Conferencia Internacional sobre Población y el Desarrollo. Nueva York, Naciones Unidas.

ONU (2014), Historia de las Naciones Unidas, consultado el 19 de noviembre de 2014, URL: http://www. un.org/es/aboutun/history/1941-1950.shtml

PNUD (2014), Índice de Desarrollo Humano Municipal en México: nueva metodología. México, Programa de las Naciones Unidas para el Desarrollo, consultado el 18 de noviembre de 2014, URL: http://www. mx.undp.org/content/dam/mexico/docs/Publicaciones/PublicacionesReduccionPobreza/InformesDesarrolloHumano/ UNDP-MX-PovRed-IDHmunicipalMexico-032014.pdf

Porras, Francisco (2011), “Javier Elguea, 2008. Razón y desarrollo. El crecimiento económico, las instituciones y la distribución de la riqueza espiritual”, en Open Insight, vol. 2, núm. 2, consultado el 12 de mayo de 2014, pp. 203-210, URL: http://openinsight.mx/wp-content/revista/OpenInsight_V2N2Resenas_Bibliograficas_p203.pdf

Rich, Adrienne (1993), “Compulsory Heterosexuality and Lesbian Existence”, en Henry Abelove, Michele Barale y David Halperin (eds.), The Lesbian and Gay Reader. New York. Routledge.

Rionda, Ramírez Jorge (2005), Contextos del desarrollo regional en México, en URL: www.eumed.net/ libros/2005/jirr/

Runeborg, Anna (2008), Sexuality: A Missing Dimension in Development. Sweden, Edita Communication AB.

Safa, Helen (1995), The Myth of the Male Breadwinner: Women and Industrialization in the Caribbean. Colorado, Westview Press.

Seers, Dudley (s/d de septiembre de 1980), Comisión Económica para América Latina y el Caribe, recuperado el 9 de julio de 2015, URL: http://repositorio.cepal.org/handle/11362/9829 
Sen, Amartya (1999), “Igualdad ¿de qué?”, en Ana María Bravo (trad.), Nuevo Examen de la Desigualdad. Madrid, Alianza Editorial.

Streeten, Paul, Shahid Javed, Mahbud U1, Norman Hicks, Frances Stewart (1986), Lo primero es lo primero. Satisfacer las necesidades humanas básicas en los países en Desarrollo. Madrid, Editorial Tecnos, S.A.

Tong, Rosemaire (1989), Feminist Thought. A Comprehensive Introduction. Colorado, Westview Press.

Valcárcel, Marcel (2006), "Génesis y evolución del concepto y enfoques sobre el desarrollo", (documento de Investigación), consultado el 12 de mayo de 2014, URL: http://www.uv.mx/mie/files/2012/10/SESION6-Marcel-Valcarcel-Desarrollo-Sesion6.pdf

Vance, Carol, (1989), "Social Construction Theory: Problems in The History of Sexuality", en A. van Kooten Nierkerk y T. van Der Meer (eds.), Homosexuality, Which Homosexuality? Amsterdam, An Dekker.

Weeks, Jeffrey (1985), Sexuality and its Discontents: Meanings, Myths and Modern Sexualities. London, Routledge y Kegan Paul.

WHO (2006), Defining sexual health. Report of a technical consultation on sexual health 28-31 January 2002, Geneva. Geneva: World Health Organization, consultado el 14 de noviembre de 2014, URL: http://www.who.int/reproductivehealth/publications/sexual_health/defining_sexual_health.pdf

World Association for Sexual Health (2014), World Sexology, consultado el 19 de noviembre de 2014, URL: http://www.worldsexology.org/wp-content/uploads/2013/08/declaracion_derechos_sexuales_sep03_2014.pdf

Fecha de recepción: 14 de abril de 2015

Fecha de aceptación: 15 de julio de 2015 